\title{
Valley-dependent current generation in nanotubes by twisted light
}

\author{
Xiaoning Zang, Nirpendra Singh, and Udo Schwingenschlögl ${ }^{*}$ \\ Physical Sciences and Engineering Division (PSE), King Abdullah University of Science and Technology (KAUST), \\ Thuwal 23955-6900, Saudi Arabia
}

(Received 31 March 2018; revised manuscript received 17 June 2018; published 1 August 2018)

\begin{abstract}
Each electronic band of a nanotube is associated with a specific crystal orbital angular momentum, in analogy to crystal momentum. We develop the semiclassical equations of motion of electrons in such twisted Bloch bands perturbed by electric and magnetic fields. These equations demonstrate that the absorption of twisted light generates valley-dependent current along the nanotube in the presence of an external inhomogeneous magnetic field. We show that the valley-dependent current provides a venue to detect valley polarization in twisted Bloch bands.
\end{abstract}

DOI: 10.1103/PhysRevB.98.085401

The extremely small reorganization energy of carbon nanotubes (CNTs) [1] makes them promising building blocks of optoelectronic circuits through which orbital angular momentum (OAM) [2-4] can be transported and processed in the form of twisted exciton wave packets [5,6]. CNTs can be mapped to graphene, a monolayer of carbon atoms with a honeycomb structure, using the lattice vector $n_{1} \vec{e}_{1}+n_{2} \vec{e}_{2}\left(\vec{e}_{1}\right.$ and $\vec{e}_{2}$ being the primitive lattice vectors) [see Fig. 1(a)], characterized by the pair of parameters $\left(n_{1}, n_{2}\right)$. By means of the helical and rotational symmetries [7], however, they can also be viewed as spirals of $N$-arm sites [see Fig. 1(b)], with $N$ being the number of arms per site (greatest common divisor of $n_{1}$ and $n_{2}$ ). As each Bloch band is thus associated with a specific integer OAM within the domain $\left[-\frac{N-1}{2}, \frac{N-1}{2}\right]$ (see Fig. 2), it is referred to as a twisted Bloch band. When $N$ is even, there is an extra Bloch band with OAM $=N / 2$ [8,9], which has no specific circularity and therefore will not be considered. On the other hand, $\sum_{n=1}^{N} e^{i 2 \pi(n-1) q / N}|n\rangle$ is the wave function of a twisted exciton with OAM $=q$ on an $N$-arm molecular ring. The phase variation $e^{i 2 \pi(n-1) q / N}$ is characteristic of twisted excitons and is responsible for the absorption and emission of twisted light [10-12]. Using the quantum current operator for a molecular ring [13], the circular current due to the eigenstates of the ring is $2 N \tau d \sin (2 \pi q / N)$, with $\tau$ being the coupling and $d$ being the distance between nearest-neighbor atoms. Therefore, excitations of twisted Bloch states of CNTs will result in a circular current, and naturally the question arises about the consequences of the force that the charge carriers experience in an external inhomogeneous magnetic field.

The transport properties of electrons in Bloch bands can be described by the semiclassical equations of motion [14]

$$
\begin{gathered}
\dot{\vec{r}}=\partial \varepsilon(\vec{k}) / \hbar \partial \vec{k}, \\
\hbar \dot{\vec{k}}=-e \vec{E}-e \dot{\vec{r}} \times \vec{B},
\end{gathered}
$$

where $\varepsilon(\vec{k})$ is the Bloch band energy, $\vec{k}$ is the momentum, $\vec{E}$ is the electric field, and $\vec{B}$ is the magnetic field. This one-band

\footnotetext{
*udo.schwingenschlogl@kaust.edu.sa
}

approximation neglects interband tunneling and therefore is only valid when the electric and magnetic fields are weak. On the other hand, for two-dimensional materials within a perpendicular magnetic field, the equations of motion of electrons in magnetic Bloch bands are given by $[15,16]$

$$
\begin{gathered}
\dot{\vec{r}}=\partial \varepsilon(\vec{k}) / \hbar \partial \vec{k}-\dot{\vec{k}} \times \Omega(\vec{k}) \hat{z}, \\
\hbar \dot{\vec{k}}=-e \vec{E}-e \dot{\vec{r}} \times \vec{B},
\end{gathered}
$$

where the magnetic field has been separated into a weak part and a strong part [17]. The second term on the right-hand side of Eq. (3) describes the effect of the strong magnetic field, with $\Omega(\vec{k})$ being the Berry curvature. The valley dependence of the Berry curvature gives rise to a valley Hall effect, which can be exploited to detect valley polarization in two-dimensional materials $[18,19]$. In this paper, we generalize this concept by developing the equations of motion of electrons in twisted Bloch bands perturbed by electric and magnetic fields. Instead of a dependence on $\vec{k}$ (due to the translational symmetry), the twisted Bloch band energies, $\varepsilon(\kappa, q)$, will depend on the quantum numbers due to the helical $(\kappa)$ and rotational $(q)$ symmetries. We will show that the $\kappa$ dependence gives rise to both a linear velocity along the CNT and a circular velocity around the CNT, whereas the $q$ dependence only gives rise to a circular velocity. The two circular velocities, not appearing in Eq. (2), result in a valley-dependent current along the CNT (assuming that the magnetic field is strong enough to dissociate the excitons), which provides a new venue to generate current and detect valley polarization in CNTs.

The dynamics of electrons in a twisted Bloch band can be described by a wave packet, i.e., a linear superposition

$$
|\Psi(t)\rangle=\int_{\kappa-\Delta \kappa / 2}^{\kappa+\Delta \kappa / 2} d \kappa C(\kappa, q, t)\left|\Psi_{\kappa, q}\right\rangle
$$

of twisted Bloch states with coefficients $C(\kappa, q, t)$, where each twisted Bloch state has the general form

$$
\left|\Psi_{\kappa, q}\right\rangle=\frac{1}{\sqrt{L N}} \sum_{l=1}^{L} \sum_{n=1}^{N} e^{i 2 \pi(l-1) \kappa / L} e^{i 2 \pi(n-1) q / N}\left|\psi_{l, n}\right\rangle,
$$




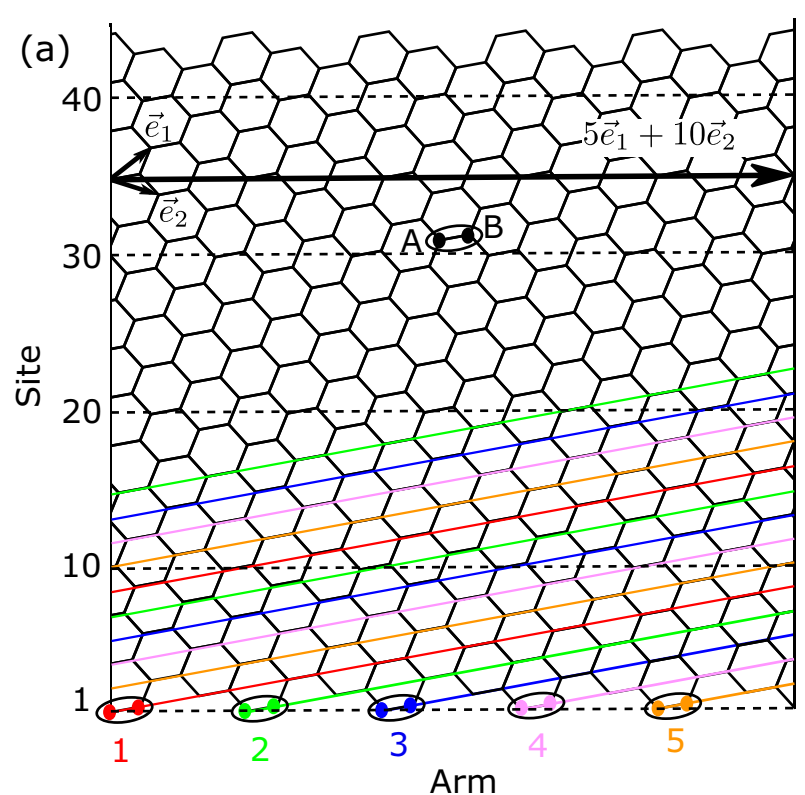

(b)
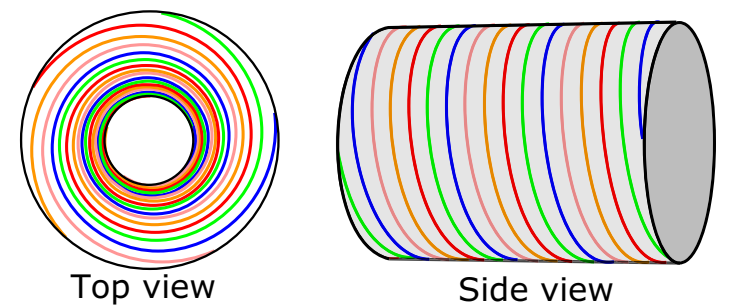

FIG. 1. (a) Mapping of the $(5,10) \mathrm{CNT}$ to graphene by means of the lattice vector $5 \vec{e}_{1}+10 \vec{e}_{2}\left(\vec{e}_{1}\right.$ and $\vec{e}_{2}$ being the primitive lattice vectors). On the other hand, the CNT can be decomposed into spirals of arms 1 (red), 2 (green), 3 (blue), 4 (pink), and 5 (orange), each consisting of two inequivalent carbon atoms, denoted as A and B. Black dashed lines indicate the five-arm sites. (b) Top and side views of the spirals.

with $L$ being the number of sites. The wave function $\left|\psi_{l, n}\right\rangle$ of the electron in arm $n$ at site $l$ is a linear combination of molecular orbitals of the two inequivalent carbon atoms of each arm; see Fig. 1(a). While physical results do not depend on the choice of quantum numbers to describe the system, choosing $\kappa$ and $q$ instead of the usual linear and angular momenta simplifies the construction of wave packets and the subsequent derivations, as the CNTs are excited by twisted light with conserved OAM. We define the helical coordinate $\vec{\chi}=z \hat{z}+r_{0} \phi_{\chi} \hat{\phi}$ as illustrated in Fig. 3. The relations

$$
z=\chi \sin (\alpha), \quad r_{0} \phi_{\chi}=\chi \cos (\alpha)
$$

indicate that the helical momentum operator, $P_{\kappa}=-i \hbar \frac{\partial}{\partial \chi}$, can be decomposed into a linear momentum operator along the $\hat{z}$ coordinate, $P_{\kappa_{z}}=P_{\kappa} / \sin (\alpha)$, and a circular momentum operator along the $\hat{\phi}$ coordinate, $P_{\kappa_{\phi}}=P_{\kappa} / \cos (\alpha)$. The wave packet defined in Eq. (5) then has a mean linear velocity of

$$
\begin{aligned}
\dot{z} & =\left\langle\Psi(t)\left|\frac{d}{d t}\left(i \frac{\partial}{\partial \kappa_{z}}\right)\right| \Psi(t)\right\rangle \\
& =\left\langle\Psi(t)\left|\frac{i}{\hbar}\left[H_{0}, i \sin (\alpha) \frac{\partial}{\partial \kappa}\right]\right| \Psi(t)\right\rangle
\end{aligned}
$$

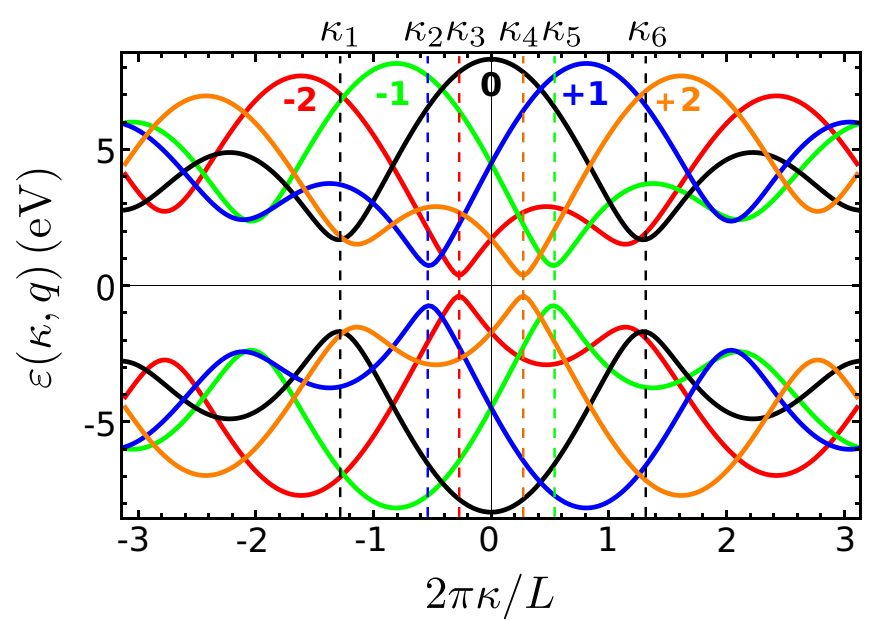

FIG. 2. Twisted Bloch bands of the $(5,10)$ CNT associated with $\mathrm{OAM}=-2$ (red), OAM $=-1$ (green), OAM $=0$ (black), OAM = 1 (blue), and OAM = 2 (orange). The band structure is calculated by a tight-binding model considering only the $p_{z}$ orbitals and nearestneighbor coupling. Dashed lines mark the band gaps.

$$
\begin{aligned}
& =\int_{\kappa-\Delta \kappa / 2}^{\kappa+\Delta \kappa / 2} d \kappa|C(\kappa, q, t)|^{2} \sin (\alpha) \frac{\partial \varepsilon(\kappa, q)}{\hbar \partial \kappa} \\
& =\sin (\alpha) \frac{\partial \varepsilon(\kappa, q)}{\hbar \partial \kappa},
\end{aligned}
$$

where $H_{0}$ is the Hamiltonian without electric and magnetic fields. In the last step, the assumption that the wave packet is narrow in $\kappa$ has been applied.

The part of the mean circular velocity originating from the helical symmetry, which is referred to as helical circular velocity in the following, can be calculated in a similar way, yielding

$$
r_{0} \dot{\phi_{\kappa}}=\cos (\alpha) \frac{\partial \varepsilon(\kappa, q)}{\hbar \partial \kappa}
$$

The part originating from the rotational symmetry, referred to as rotational circular velocity in the following, however, is not well defined in terms of $\frac{\partial}{\partial q}$ because of the discontinuity of $q$ space. Instead, the circular momentum operator, $P_{q}=-i \hbar \frac{\partial}{r_{0} \partial \phi_{q}}$, gives

$$
\begin{aligned}
r_{0} \dot{\phi}_{q} & =\left\langle\Psi(t)\left|P_{q} / m\right| \Psi(t)\right\rangle \\
& =\left\langle\Psi_{\kappa, q}\left|-i \hbar \frac{\partial}{r_{0} m \partial \phi_{q}}\right| \Psi_{\kappa, q}\right\rangle .
\end{aligned}
$$

Electric and magnetic fields do not affect Eqs. (8)-(10), but solely determine the dynamic equations for $\kappa$. Considering a homogeneous electric field along the CNT, $H^{\prime}=-e \Phi(z)$, we have

$$
\begin{aligned}
\hbar \dot{\kappa}_{z} \hat{z} & =\frac{i}{\hbar}\left\langle\Psi(t)\left|\left[H_{0}-e \Phi(z), P_{\kappa_{z}}\right]\right| \Psi(t)\right\rangle \\
& =-e\left\langle\Psi(t)\left|\left[\Phi(z), \frac{\partial}{\partial z}\right]\right| \Psi(t)\right\rangle=-e \vec{E} .
\end{aligned}
$$

Modeling the external inhomogeneous magnetic field by a radial magnetic field of the form $\vec{B}=B r \hat{r}$, which is generated by the vector potential $\vec{A}=-B r z \hat{\phi}$, we have $H^{\prime}=e B r z P_{\phi} / m$ with $P_{\phi}=P_{\kappa_{\phi}}+P_{q}$. Since $H^{\prime}$ commutes 


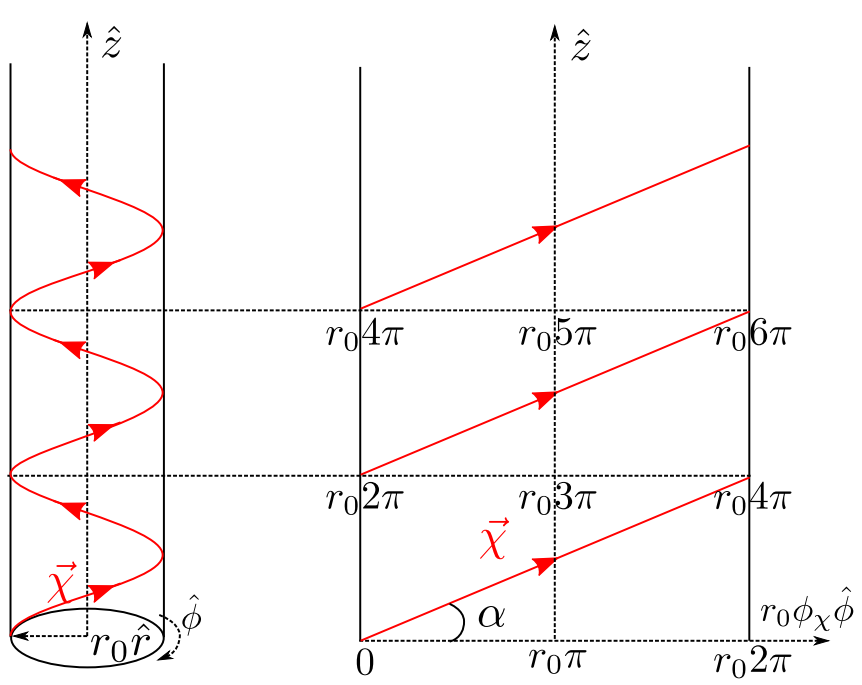

FIG. 3. Definition of the helical coordinate $\vec{\chi}=z \hat{z}+r_{0} \phi_{\chi} \hat{\phi}$ in cylindrical coordinates (left) and unfolded into the $\phi z$ plane (right).

with $P_{\phi}$ but not with $P_{K_{z}}$, we have $\dot{P}_{\phi}=0$ and

$$
\begin{aligned}
\hbar \dot{\kappa}_{z} \hat{z} & =\frac{i}{\hbar}\left\langle\Psi(t)\left|\left[H^{\prime}, P_{\kappa_{z}}\right]\right| \Psi(t)\right\rangle=-e\left\langle\Psi(t)\left|\operatorname{Br} P_{\phi} / m\right| \Psi(t)\right\rangle \\
& =-e B r_{0}\left|\vec{v}_{\phi}\right| \hat{z}=-e \vec{v}_{\phi} \times \vec{B},
\end{aligned}
$$

where $\vec{v}_{\phi}=\left(r_{0} \dot{\phi}_{\kappa}+r_{0} \dot{\phi}_{q}\right) \hat{\phi}$ and it is assumed that the magnetic field at the wall of the CNT is $B r_{0}$. The dynamics of electrons in twisted Bloch bands is thus given by

$$
\begin{gathered}
\dot{z} \hat{z}+r_{0} \dot{\phi} \hat{\phi}=[\sin (\alpha) \hat{z}+\cos (\alpha) \hat{\phi}] \frac{\partial \varepsilon(\kappa, q)}{\hbar \partial \kappa}+r_{0} \dot{\phi}_{q} \hat{\phi}, \\
\hbar \dot{\kappa}_{z} \hat{z}=-e \vec{E}-e \vec{v}_{\phi} \times \vec{B} .
\end{gathered}
$$

Equation (13) resembles Eq. (1) except for the extra velocity term around the CNT. Equation (14) resembles Eqs. (2) and (4), but $\vec{v}_{\phi}$ is a circular velocity and the magnetic-field points along the $\hat{r}$ coordinate.

A direct consequence of the circular velocity term in Eq. (14) is current generation when the CNT is illuminated by twisted light and exposed to a magnetic field with a radial

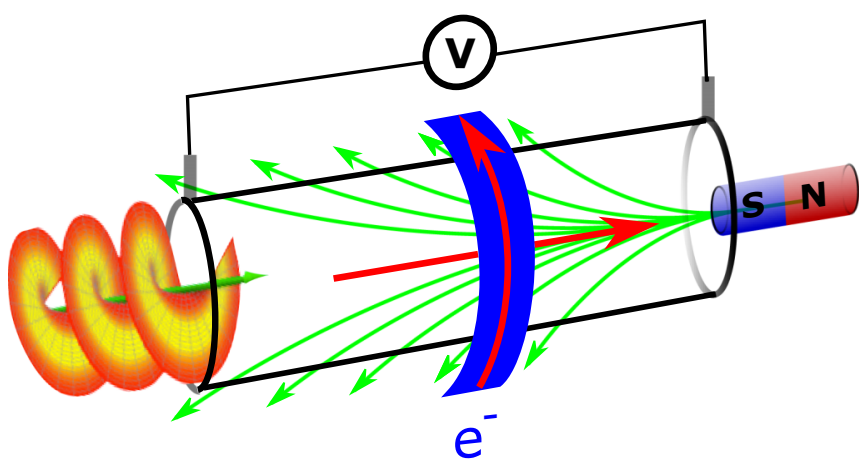

FIG. 4. Setup for the measurement of a valley-dependent current. A magnetic field with a radial component is provided by the magnet on the right side of the CNT and a twisted light beam is applied from the left side of the CNT. The measured voltage depends on the OAM of the twisted light.
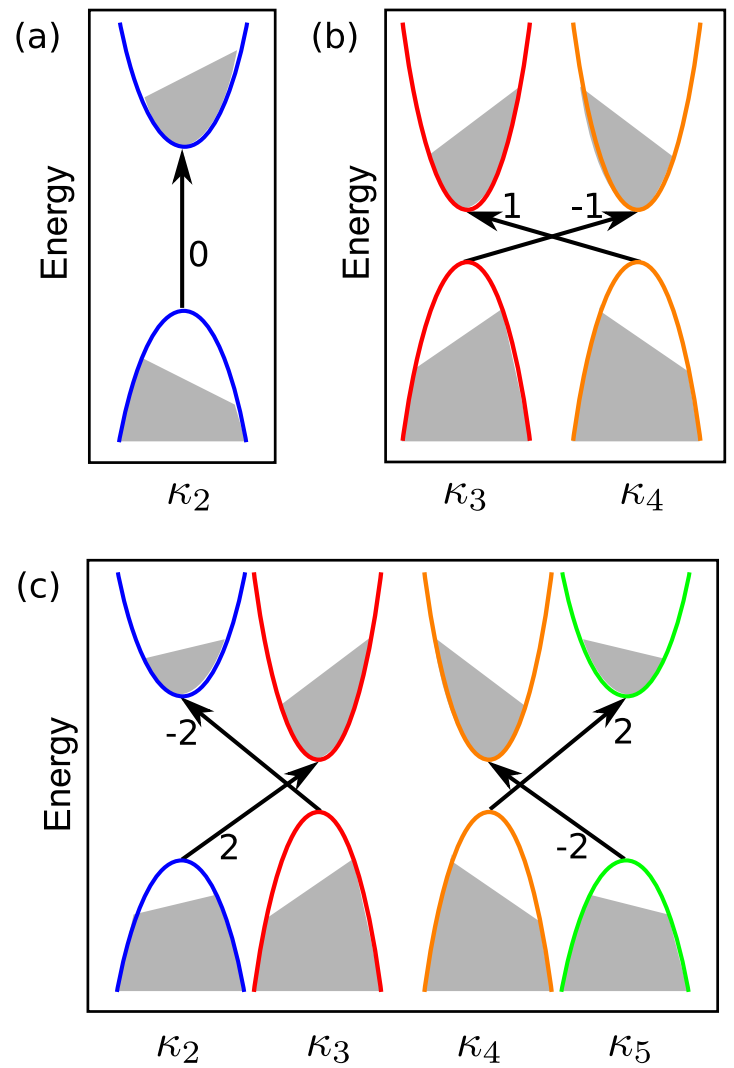

FIG. 5. Excitation in twisted Bloch bands by (a) twisted light with $\mathrm{OAM}=0$ in an electric field, (b) twisted light with OAM $=1$ or -1 in a magnetic field with radial component, and (c) twisted light with $\mathrm{OAM}=2$ or -2 in a magnetic field with a radial component. The valleys $\kappa_{2}, \kappa_{3}, \kappa_{4}$, and $\kappa_{5}$ are defined in Fig. 2. The shaded areas represent the distribution function $f(\kappa)$.

component, as shown in Fig. 4. To demonstrate this new mechanism of current generation, we employ Boltzmann theory. The distribution function in the relaxation-time approximation is

$$
f(\kappa)=f_{0}(\kappa)+\frac{e}{\hbar} \tau(\kappa)\left(\vec{E}+\vec{v}_{\phi} \times \vec{B}\right) \cdot \nabla_{\kappa} f_{0}(\kappa),
$$

where $f_{0}(\kappa)$ is the Fermi distribution function and $\tau$ is the relaxation time. Then the current along the CNT is given by

$$
J_{z}=-e \int d \kappa f(\kappa) \dot{z}(\kappa)
$$

$J_{z}=0$ without electric and magnetic fields, since $f(\kappa)$ is symmetric and $\dot{z}(\kappa)$ is antisymmetric around the valleys (see $\kappa_{2}, \kappa_{3}, \kappa_{4}$, and $\kappa_{5}$ in Fig. 2). There will be a net current when an electric field is applied along the CNT (without magnetic field), since now $f(\kappa)$ is no longer symmetric around the valleys [see Fig. 5(a)] for any OAM of the twisted light. Electrons in conventional Bloch bands will have the same distribution function and therefore result in a net current, i.e., nothing is special for electrons in twisted Bloch bands so far. New physics is introduced when a magnetic field with a radial component couples to the velocity; see Eq. (15).

Using Eqs. (8) and (9), we examine how the linear velocity and the helical circular velocity vary with $\kappa$. As Eq. (10) tells us only how to calculate the rotational circular velocity, we apply a tight-binding model restricted to coupling between the 


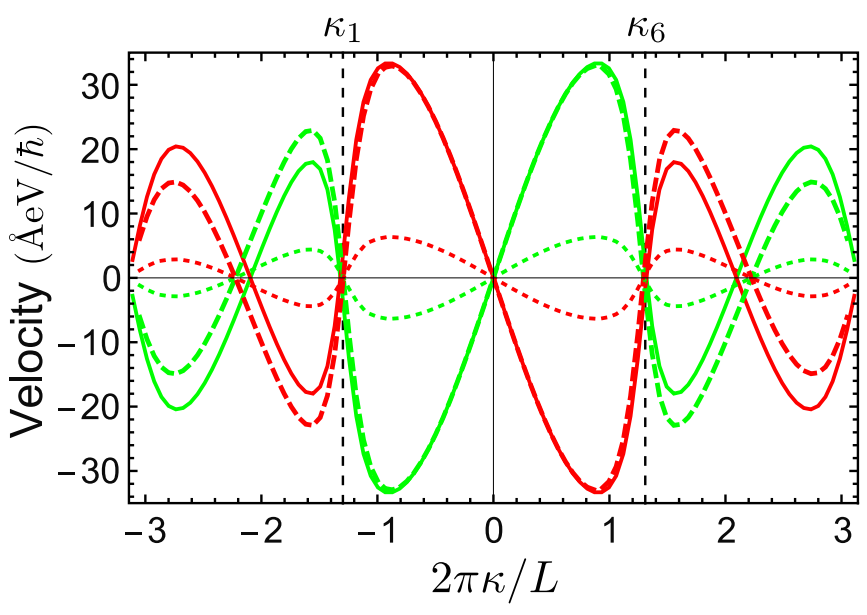

FIG. 6. Velocity along the CNT (dashed curves), helical circular velocity (dotted curves), and rotational circular velocity (solid curves) for $\mathrm{OAM}=0$. Green refers to the valence band, red to the conduction band.

$p_{z}$ orbitals of nearest-neighbor carbon atoms,

$$
H_{0}=\sum_{\left\langle l, n ; l^{\prime}, n^{\prime}\right\rangle} \tau a_{l, n}^{A \dagger} a_{l^{\prime}, n^{\prime}}^{B}+\text { H.c., }
$$

where $\left\langle l, n ; l^{\prime}, n^{\prime}\right\rangle$ indicates nearest neighbors, $a_{l, i}^{A \dagger}$ is the creation operator for an electron on carbon atom $A$ in arm $i$ at site $l, a_{l^{\prime}, n^{\prime}}^{B}$ is the annihilation operator for an electron on carbon atom $B$ in arm $n^{\prime}$ at site $l^{\prime}$, and the coupling $\tau$ is set to a value of $-2.77 \mathrm{eV}$ [20]. The velocity operator can be derived from the polarization operator [21]

$$
\vec{P}=\sum_{l, n}\left(\vec{R}_{l, n}^{A} a_{l, n}^{A \dagger} a_{l, n}^{A}+\vec{R}_{l, n}^{B} a_{l, n}^{B \dagger} a_{l, n}^{B}\right)
$$

as

$$
\begin{aligned}
\vec{v}= & \frac{\partial \vec{P}}{\partial t}=-i\left[H_{0}, \vec{P}\right] / \hbar \\
= & -\frac{i \tau}{\hbar} \sum_{\left\langle l, n ; l^{\prime}, n^{\prime}\right\rangle}\left[\left(\vec{R}_{l, n}^{A}-\vec{R}_{l^{\prime}, n^{\prime}}^{B}\right) a_{l^{\prime}, n^{\prime}}^{B \dagger} a_{l, n}^{A}\right. \\
& \left.+\left(\vec{R}_{l, n}^{B}-\vec{R}_{l^{\prime}, n^{\prime}}^{A}\right) a_{l^{\prime}, n^{\prime}}^{A \dagger} a_{l, n}^{B}\right],
\end{aligned}
$$

where $\vec{R}$ denotes the positions of the carbon atoms, taking the carbon-carbon bond length as $1.44 \AA$ [20]. It turns out that the same linear velocity and the same helical circular velocity are obtained from Eq. (19) and from Eqs. (8) and (9), which verifies the validity of Eq. (19) for calculating the rotational circular velocity. Figure 6 shows the results obtained from Eq. (19) for the conduction and valence bands with $\mathrm{OAM}=0$. The linear velocity and the helical circular velocity are zero when $\partial \varepsilon(\kappa) / \partial \kappa=0$, as expected. The zeros of the rotational circular velocity, however, are shifted, which, as will be explained later, is the source of net current generation when the CNT is exposed to a magnetic field with a radial component. More specifically, for the conduction band the zeros shift left (right) at the band minimum and right (left) at the band maximum for $\kappa>0(\kappa<$ $0)$, and in the opposite directions for the valence band. The shift gets larger as $|\kappa|$ grows. Note that these shifts apply to all the bands in Fig. 2. With the rotational circular velocity at hand, we calculate $f(\kappa)$ and show in Fig. 7 results for different values of $B$, assuming $\tau=5$ ps [22]. The curves for $B \neq 0$ are not

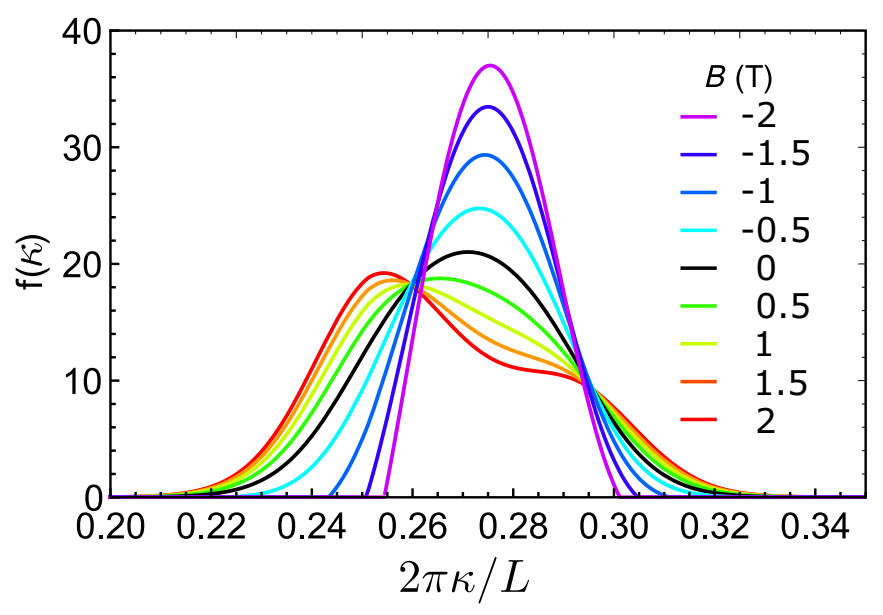

FIG. 7. Distribution function for an electron excited into the conduction-band valley at $\kappa_{4}$.

symmetric due to the different symmetries of the helical and rotational circular velocities.

Assume that $\varepsilon(\kappa)$ is symmetric at the valleys shown in Fig. 2. Then the linear velocity and the helical circular velocity would be antisymmetric at the valleys, i.e., they cannot break the symmetry of $f(\kappa)$ and therefore cannot introduce any net current. The rotational circular velocity, however, is not symmetric at the valleys, such that $f(\kappa)$ is not symmetric. Figures 5(b) and 5(c) indicate that opposite current will be generated and, consequently, opposite potentials can be measured across the setup of Fig. 4 when twisted light with opposite OAM is applied. In Fig. 5(c) the tilts of the shaded areas illustrate the effects of the shifts of the zeros of the rotational circular velocity at the different valleys. Assuming that the same amount of electrons are excited at each valley, the excitation by twisted light with OAM $=-2$, for example, will generate a smaller current than the excitation by twisted light with $\mathrm{OAM}=-1$. Excitation by twisted light with $\mathrm{OAM}=0$ will generate no net current, as the electrons in the valence and conduction bands will be moving in opposite directions. We note that the valley-dependent current, therefore, makes it possible to detect the valley polarization in the twisted

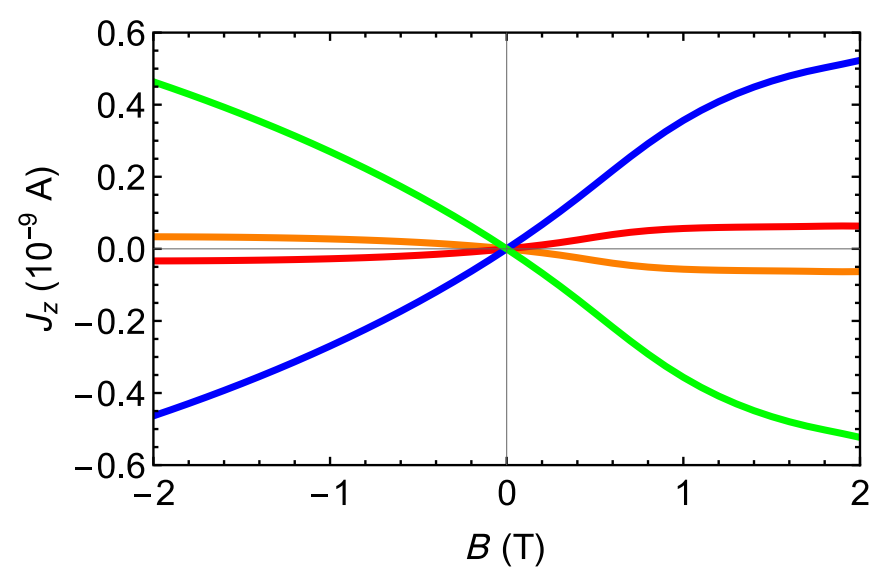

FIG. 8. Current along the CNT generated by twisted light with OAM $=-2$ (red), OAM $=-1$ (green), OAM = 1 (blue), and $\mathrm{OAM}=2$ (orange). 
Bloch bands of CNTs by means of a magnetic field with a radial component. Figure 8 gives numerical results for $J_{z}$ obtained from Eq. (16), showing that observable currents can be generated by reasonable magnetic fields.

In conclusion, the semiclassical equations of motion of electrons in twisted Bloch bands have been derived, which has enabled us to analyze the transport properties by means of Boltzmann theory. Generation of valley-dependent current is predicted when a CNT is exposed to an external inhomogeneous magnetic field and excited by twisted light with $\mathrm{OAM} \neq 0$. All excitations generating valley-dependent current are indirect, which requires phonons to be involved in order to compensate for the difference in linear momentum and
OAM between the valleys. However, it turns out that the valleys approach each other for growing $N$. The extremely low reorganization energy of CNTs, which is reported to be on the order of tens of meV [1], makes it possible to keep the moving electrons coherent and thus the OAM constant. The introduced mechanism of current generation can be used to detect valley polarization in the twisted Bloch bands of CNTs. While we have used CNTs to demonstrate our ideas, the derived conclusions apply to nanotubes in general.

The research reported in this publication was supported by funding from King Abdullah University of Science and Technology (KAUST).
[1] R. Ihly, K. S. Mistry, A. J. Ferguson, T. T. Clikeman, B. W. Larson, O. Reid, O. V. Boltalina, S. H. Strauss, G. Rumbles, and J. L. Blackburn, Tuning the driving force for exciton dissociation in single-walled carbon nanotube heterojunctions, Nat. Chem. 8, 603 (2016).

[2] H. Ajiki and T. Ando, Electronic states of carbon nanotubes, J. Phys. Soc. Jpn. 62, 1255 (1993).

[3] E. D. Minot, Y. Yaish, V. Sazonova, and P. L. McEuen, Determination of electron orbital magnetic moments in carbon nanotubes, Nature (London) 428, 536 (2004).

[4] E. A. Laird, F. Kuemmeth, G. A. Steele, K. Grove-Rasmussen, J. Nygård, K. Flensberg, and L. P. Kouwenhoven, Quantum transport in carbon nanotubes, Rev. Mod. Phys. 87, 703 (2015).

[5] X. Zang and M. T. Lusk, Twisted molecular excitons as mediators for changing the angular momentum of light, Phys. Rev. A 96, 013819 (2017).

[6] X. Zang and M. T. Lusk, Angular momentum transport with twisted exciton wave packets, Phys. Rev. B 96, 155104 (2017).

[7] C. T. White, D. H. Robertson, and J. W. Mintmire, Helical and rotational symmetries of nanoscale graphitic tubules, Phys. Rev. B 47, 5485 (1993).

[8] M. Damnjanović and M. Vujičić, Magnetic line groups, Phys. Rev. B 25, 6987 (1982).

[9] M. Damnjanović and I. Milošević, Line Groups in Physics (Springer, Boston, 2010).

[10] L. Allen, M. W. Beijersbergen, R. J. C. Spreeuw, and J. P. Woerdman, Orbital angular momentum of light and the transformation of Laguerre-Gaussian laser modes, Phys. Rev. A 45, 8185 (1992).
[11] Q. Zhan, Properties of circularly polarized vortex beams, Opt. Lett. 31, 867 (2006).

[12] M. D. Williams, M. M. Coles, K. Saadi, D. S. Bradshaw, and D. L. Andrews, Optical Vortex Generation from Molecular Chromophore Arrays, Phys. Rev. Lett. 111, 153603 (2013).

[13] G. D. Mahan, Many-particle Physics (Springer, Boston, 2000).

[14] N. W. Ashcroft and N. D. Mermin, Solid State Physics (Saunders, Philadelphia, 1976).

[15] M.-C. Chang and Q. Niu, Berry Phase, Hyperorbits, and the Hofstadter Spectrum, Phys. Rev. Lett. 75, 1348 (1995).

[16] G. Sundaram and Q. Niu, Wave-packet dynamics in slowly perturbed crystals: Gradient corrections and Berry-phase effects, Phys. Rev. B 59, 14915 (1999).

[17] M.-C. Chang and Q. Niu, Berry phase, hyperorbits, and the Hofstadter spectrum: Semiclassical dynamics in magnetic Bloch bands, Phys. Rev. B 53, 7010 (1996).

[18] D. Xiao, W. Yao, and Q. Niu, Valley-Contrasting Physics in Graphene: Magnetic Moment and Topological Transport, Phys. Rev. Lett. 99, 236809 (2007).

[19] J. R. Schaibley, H. Yu, G. Clark, P. Rivera, J. S. Ross, K. L. Seyler, W. Yao, and X. Xu, Valleytronics in 2D materials, Nat. Rev. Mater. 1, 16055 (2016).

[20] J. Mintmire and C. White, Electronic and structural properties of carbon nanotubes, Carbon 33, 893 (1995).

[21] D. Cabib and T. A. Kaplan, On the definition of the current operator application to the Hubbard model, Phys. Status Solidi B 58, 85 (1973).

[22] M. Lazzeri and F. Mauri, Coupled dynamics of electrons and phonons in metallic nanotubes: Current saturation from hotphonon generation, Phys. Rev. B 73, 165419 (2006). 